psychosis and unnecessary discontinuation in our cases led to relapses and further difficulties in management. Reintroduction of psychotropic medication decreased the incidences of relapses and confirmed the safety of neuroleptics in Gilbert's syndrome as suggested by others (Cohen \& Cohen, 1992; Durst et al, 1993).

Psychiatric illnesses are regarded as complex disorders: disorders which show evidence of gene effect but do not show clear Mendelian patterns of inheritance. Comorbidity of such illneses with other genetic disorders might be of help in understanding their psychopathology. A hypothetical explanation for the higher incidence of hyperbilirubinaemia and schizophrenia, genetic predisposition, was emphasised by Muller et al (1991). Further reporting of similar cases to the present study may emphasise the significant comorbidity of Gilbert's syndrome and mental disorder which will need further clarification.

\section{References}

Davidson. A. R., Rojas-Beuno, A.. Thompson, R. P. H., et al (1975) Reduced caloric intake of nicotinic acid provocation tests in the diagnosis of Gilbert's syndrome. British Medical Joumal. ii. 480.
Blanckaert. N. \& FeVery. J. (1990) Physiology and pathophysiology of bilirubin metabolism. In Hepatology: A Textbook of Liver Disease (eds T. Zakim \& T. D. Boyer) pp. 254-285. Philadelphia. PA: W. B. Saunders.

COHEN. L. S. \& COHEN, D. E. (1992) Lithium induced hyperbilirubinaemia in an adolescent. Journal of Clinical Psychopharmacology, 11, 274-275.

DURST, R., JABTINSKY-RUBIN. K.. DOREVITCH. A. et al (1993) Idiopathic unconjugated hyperbilirubinaemia (Gilbert's syndrome) and concurrent psychotropic drug administration. Pharmacopsychiatry. 26. 49-52.

GILBERT, A. \& LEREBOULLET, P. (1901) La cholemie simple familiale. Semaine Médicale, 21, 241-245.

LOYD, D. W.. TsuAnG, M. T. \& BENGE, J. W. (1982) A study of a family with Leopard syndrome. Journal of Clinical Psychiatry. 43, 113-116.

Muller, N., SCHILleR, P. \& ACKenheIl, M. (1991) Coincidence of schizophrenia and hyperbilirubinaemia. Pharmacopsychiatry. 24. 225-228.

Seig, A.. Stich, A. RAEDSCH, R., et al (1986) Gilbert's syndrome: diagnosis by typical serum bilirubin pattern. Clinical Chimica Acta, 184, 41-48.

TAnoR, S. (1984) Gilbert's syndrome as a cause of postoperative jaundice. Anaesthesia. 39. 1222-1224.

*A. S. Mahdi, Senior House Officer in Psychiatry. and M. S. Elameer, Consultant Psychiatrist, St David's Hospital, Carmarthen. Carmarthenshire. SA31 3HB

*Correspondence

\title{
Butterflies, fractals and psychiatry
}

\author{
Steven Reid
}

In this paper concepts related to the field of non-linear dynamics and choos theory are discussed, and their relevance to psychiatry is considered.

'In all chaos there is a cosmos, in all disorder a secret order' (Jung, 1954)

For many, chaos theory is a new branch of science that appears to involve the butterfly effect' and 'fractals', but which is little understood and has no obvious relevance to psychiatry. The purpose of this paper is to unravel a number of unfamiliar concepts, and describe some of the influences this emergent mathematical theory has had upon medicine and more specifically, psychiatry.
Previously the application of basic sciences to clinical medicine has relied upon linear, deterministic processes which were relatively easily understood and predictable. Classical determinism as propounded by Laplace, viewed the universe as a gigantic, intricate clockwork machine in which all events were predictable and the magnitude of any evoked response was proportionate to the size of its stimulus. However, there are physical systems which are seemingly non-linear and highly unpredictable such as turbulence in liquids (e.g. blood flow in the heart) and cerebral electrical activity. In these situations small changes can result in large and unanticipated effects. Since the 1960s, scientists from a variety of backgrounds have been attempting to provide models for this 
unpredictable and complex behaviour. A meteorologist, Lorenz (1986) attempting to model global weather systems, noted the degree to which the components of a non-linear system interact, thereby producing a series of feedbacks (both positive and negative) which can lead to a tremendous variation in consequences. This explains the difficulty in long-range weather forecasting, and led to the coining of the term 'the butterfly effect' (originating from the title of his address to the American Association for the Advancement of Science in 1979, 'Predictability: does the flap of a butterfly's wings in Brazil set off a tornado in Texas?'). This potential for such an exaggerated range of outcomes is known as 'sensitive dependence on initial conditions'. This area of study has expanded to become nonlinear dynamics and has been applied to fields as diverse as meteorology, economics (with attempts to predict the rise and fall of cotton prices), car manufacture and medicine. All of these non-linear processes resist any form of reductionist analysis but a common theme is the potential for striking, unexpected effects arising from minimal changes in initial variables. One such potential effect is the much vaunted phenomenon, chaos.

Chaos theory, although pioneered by Lorenz, was given its name in 1972 by the mathematician James Yorke. Chaos refers to a seemingly random type of variability that can arise from a non-linear system. Despite its connotations, in this context chaos is not completely unfettered, random behaviour. It has the characteristic of appearing random and is unpredictable at certain scales, yet retains an overall degree of organisation which can be modelled. Many previously observed phenomena and patterns of behaviour, thought to be unpredictable and unexplainable, are now considered to operate on non-linear principles. This implies a potential for greater understanding of these processes.

The term fractal (derived from the Latin, fractus, meaning broken) was introduced by a French mathematician. Benoit Mandelbrot, in 1975 (Peitgen \& Richter, 1986) and is related to chaos. A fractal is an object consisting of a pattern that when magnified, reveals repetitive levels of detail so that a similar structure exists on all scales. This property is known as selfsimilarity. Mandelbroit found fractals everywhere - coastlines, trees, forked lightening, cauliflower florets. Fractal structures abound in medicine e.g. the arterial and venous circulatory systems, the central nervous sytem, the tracheobronchial tree. Possessing self-similarity offers these systems a great advantage: they are able to contain a vast surface area in a limited volume. Fractals are also evident in complex processes with a timescale e.g. a graph of a series of fluctuations in respiration over time would reveal similar irregularities whether viewed on a scale of $1 \mathrm{~min}$ or $100 \mathrm{~min}$. Other examples include heartrate variability, systemic blood pressure and variation in white cell counts. This fractal or scale-free organisation seems to be a central principle of physiological structure and function. It would therefore be a reasonable assumption that with pathology there may be an associated breakdown of the fractal process which would result in a fractal-free system dominated by one scale. A system dominated by one scale is by definition periodic and the appearance of highly periodic events in disease is wellrecognised e.g. periodic tremors in neurological conditions, periodic fevers in Hodgkin's lymphoma and periodic electroencephalogram rhythms during a seizure. It has been maintained on this basis that the normally functioning heart shows more chaotic behaviour than the diseased one. This seemingly paradoxical conclusion is at odds with the traditional, 'common sense' view that the collapse of the rhythms of a healthy organism is at the root of many physiological disorders.

The role of non-linear dynamics in clinical medicine has only recently begun to be examined. but workers in the field are optimistic. As early as in the 1920s, two Dutch physiologists, van der Pol and van der Mark had shown that by using logistic equations (which are of fundamental importance in non-linear theory), in an attempt to formulate a mathematical model of cardiac pathology, they could generate cardiac arrhythmias (Mackay \& Glass, 1977). The arrhythmias were precipitated by abrupt non-linear transitions known as bifurcations. A bifurcation is a descriptive term for the situation in which a very small change in the value of a variable affecting a system causes it to change abruptly from one type of behaviour to another. Within cardiovascular medicine there is an expectation of a number of practical applications in the shortterm (Goldberger, 1996). The use of non-linear dynamics in physiological monitoring has already shown promise in predicting those at rick of electrophysiological instability such as the automatic detection of the onset of pathological changes in heart-rate beat-to-beat variability, which may be a precursor to ventricular arrhythmias and sudden cardiac death. Other potential sites of application for chaos theory include the detection of subtle ST-T alternans (alternating amplitude from beat-tobeat on an electrocardiogram) and the detection of a breakdown in fractal scaling also leading to arrhythmias. With a greater understanding of the origin of arrhythmias another possible development involves the refinement of cardiac defibrillators. Previously the design of defibrillators, such as the size and shape of the electrical charge, has been empirically based. Principles of non-linear dynamics may provide a theoretical 
basis for improving their efficiency and thereby success rate.

Applications of non-linear dynamics have yet to make a significant impact upon clinical psychiatric practice. They have been useful in developing models for research e.g. Hubermans' simulation of the scanning abnormalities in the eye movements of patients with schizophrenia and their relatives (Gleick, 1988). More recently. Paulus et al (1996) have used non-linear theory in their analysis of people with schizophrenia being tested by their responses to a simple choice task. They were able to demonstrate a significantly greater degree of perseveration and unpredictability compared with controls, and were able to quantify the degree of behavioural disorganisation.

Work by Gottschalk et al (1995) has highlighted more practical implications of non-linear dynamics. They introduced principles of chaos theory in their study of a group of patients with rapid-cycling bipolar affective disorder as a means of analysing the temporal variation in their mood. Pre-existing models of mood variation in this disorder consisted of (a) aberrant biological rhythms, based on short-term, intensive monitoring of mood cycles in which there is a marked tendency for periods of mania to follow periods of depression, and (b) the kindling model, in which episodes of mood disorder become more frequent as the disorder persists and later episodes seem to arise spontaneously rather than being related to precipitants. Both of these models fail to explain the irregular fluctuation of mood which is often observed in the clinical situation. Gottschalk et al found that the mood variation in this group of patients showed a chaotic pattern, without true periodicity or progressively accelerating course, and interestingly this pattern had a degree of organisation and predictability that was absent from the euthymic controls. This is comparable with electroencephalogram wave patterns in epilepsy-abnormality being associated with order. This chaotic model also offers a coherent explanation of the role of psychosocial stressors in bipolar disorder. An appropriate timed stimulus (stressor) of sufficient amplitude may exacerbate symptoms by driving a vulnerable system into sustained chaotic dynamics. In this way a stressor may produce long-lasting effects, with subsequent episodes apparently unlinked to any precipitant, which may explain why later episodes in bipolar disorder appear to arise spontaneously. The high level of organisation of these patients' moods suggests the possibility of predicting the pathway of affective change and developing a pulsed or phasic treatment which could be used in anticipation. Unfortunately, this study was small (using seven bipolar patients) and warrants replication on a larger scale but the authors do demonstrate that both biomedical and psychological contributors to mood and behaviour can be employed in nonlinear models.

Non-linear dynamics has some determined advocates. Mandell (1985), a US psychiatrist criticised previous psychiatric practice for being narrow-minded and upsophisticated . . . "psychiatry is soon to be faced with a radical paradigmatic shift in neuropsychobiology that may have far-reaching implications for pathophysiology and treatment. Current paradigms of medical research will collapse because of their failure to deal with diseases that emerge autonomously". This comment would appear to be somewhat overblown, but chaos theory clearly does offer solutions to several problems in contemporary physiology and biology. The likely impact of non-linear dynamics upon psychiatry remains unclear at present, but its potential value in elucidating the mechanisms of mental illness merits continuing investigation.

\section{References}

GLEICK, J. (1988) Chaos. London: Heinemann.

GOLDBERGER, A. L. (1996) Non-linear dynamics for clinicians. Lancet, 347, 1312-1314.

GotTSChalk, A., BAuER, M. S. \& WhYBRow, B. C. (1995) Evidence of chaotic mood variation in bipolar disorder. Archives of General Psychiatry, 52, 947-959.

JuNG, C. G. (1954) The Development of Personality. London: Routledge.

LORENZ, E. N. (1986) Large-scale motions of the atmosphere. In Advances in Earth Science (ed. P. M. Hurley), pp. 95-109. Cambridge, MA: MIT Press.

MACKAY, M. \& GLASS, L. (1977) Oscillation and chaos in physiological control systems. Science, 197, 287.

MANDELL, A. J. (1985) From molecular biological simplification to more realistic central nervous system dynamics: an opinion. In Psychobiological Foundations of Clinical Psychiatry (ed. O. Cavenar), pp. 361-366. New York: Lippincott.

Paulus, M. P., GeYer, M. A. \& BRAFf, D. L. (1996) Use of methods from chaos theory to quantify a fundamental dysfunction in the behavioural organisation of schizophrenic patients. American Journal of Psychiatry. 163. 714-717.

PEITGEN, H. \& RichteR, P. (1986) The Beauty of Fractals. Berlin: Springer-Verlag.

Steven Reid, Senior Registrar in General Psychiatry, Springfield Hospital, 61 Glenburnie Road, London SW17 7DJ 